\title{
Fitness and body composition profiling of elite junior South African rugby players
}

\author{
$J^{\text {Durandt }}{ }^{1}$ (BSc (Med) (Hons) Exercise Science (Biokinetics)) \\ $S$ du Toit $^{1}$ (BA (Hons) Biokinetics) \\ $J_{\text {Borresen }}^{2}$ (BSc (Med) (Hons) Exercise Science (Biokinetics)) \\ T Hew-Butler ${ }^{2}$ (DPM) \\ H Masimla ${ }^{3}$ (BA (Phys Ed)) \\ I Jakoet ${ }^{3}$ (MB ChB, MSc (Sports Medicine)) \\ $M$ Lambert $^{2}$ (PhD) \\ ${ }^{1}$ Discovery Health High Performance Centre, Sports Science Institute of South Africa, Newlands, Cape Town \\ ${ }^{2}$ UCT/MRC Research Unit for Exercise Science and Sports Medicine, Department of Human Biology, Faculty of Health Sciences, University \\ of Cape Town \\ ${ }^{3}$ South African Rugby Union, Newlands, Cape Town
}

\begin{abstract}
Objective. The aim of this study was to describe the body composition, strength and speed characteristics of elite junior South African rugby players.

Design. Cross-sectional.

Setting. Field study.

Subjects. Rugby players (16 and 18 years old, $N=174$ ) selected for the South African Rugby Union National Green Squad.

Outcome measures. Body composition, $10 \mathrm{~m}$ and $40 \mathrm{~m}$ speed, agility, 1RM bench press, underhand pull-ups, push-ups, multistage shuttle run.

Results. The under-16 players were on average shorter $(175.6 \pm 5.7$ v. $179.2 \pm 6.7 \mathrm{~cm})$, weighed less $(76.5 \pm 8.2$ v. $84.8 \pm 8.3 \mathrm{~kg}$ ) had less upper body absolute strength $(77.1 \pm 11.8 \mathrm{~kg}$ v. $95.3 \pm 16.7 \mathrm{~kg})$ and muscular endurance ( $41 \pm 12$ v. $52 \pm 15$ push-ups) and aerobic fitness (87.1 \pm 19.4 v. $93.5 \pm 15.3$ shuttles) than the under-18 players. There were no differences in body fat, sprinting speed
\end{abstract}

\section{CORRESPONDENCE:}

M Lambert

UCT/MRC Research Unit for Exercise Science and Sports Medicine

PO Box 115

Newlands 7725

Cape Town, South Africa

Tel: $\quad$ 021-650 4558

Fax: $\quad$ 021-686 7530

E-mail: mlambert@sports.uct.ac.za
(10 $\mathrm{m}$ and $40 \mathrm{~m}$ ) or agility between the two age groups. There were differences between playing positions, with the props having the most body fat, strongest upper bodies, slowest sprinting speed, least agility and lowest aerobic capacity compared with players in the other positions.

Conclusion. This study provides data for elite junior rugby players and can be used to monitor the progression of players after intervention while also assisting with talent identification for the different playing positions.

\section{Introduction}

Many studies have examined the physical characteristics of elite, ${ }^{3,9}$ amateur, ${ }^{16,19}$ adolescent ${ }^{6,18,19}$ and pre-adolescent rugby players. ${ }^{17}$ A majority of these analyses divide players into two distinct categories - forwards and backs, based on unique physiological criteria specific to these positions. ${ }^{3,6,16,19}$ Information derived from these studies may be used to identify superior rugby players and also guides conditioning strategies that may enhance team and individual performance.

The South African Rugby Union Green Squad is a selection of elite junior players who represent the next generation of national team talent. The goal of the Green Squad programme is to identify and develop rugby talent with a long-term vision of channelling these players into the national squad. As rugby is a highly demanding physical, tactical and skill-based team sport, ${ }^{9}$ substantial resources and emphasis should be directed towards developing and maintaining physical fitness in players from an early age. ${ }^{10}$ In accordance with this, the objective of the study was to provide a descriptive profile of the under-16 and under-18 year elite junior players. Specifically, the aim was to highlight the anthropometric, strength and speed differences between the 9 categories of playing positions and between the 2 age 
groups with the intention of using the data for future talent identification and training intervention projects. This study is novel, as no such data exist on junior rugby players.

\section{Methods}

Rugby players $(N=174)$ selected for the South African Rugby Union National Green Squad in 2003 were used in this study. The players were selected from 14 provincial under-16 teams which competed at the National Grant Komo tournament and 14 provincial under-18 teams which competed at the annual under-18 national Craven Week tournament. Players were selected from these provincial teams by a national panel appointed by the South African Rugby Union. The players were divided into 16-year-old $(N=92)$ and 18-year-old groups $(N=$ 82). The study was cleared by the University of Cape Town Research and Ethics Committee. Players gave their consent to participate and an informed consent form was signed by the parent/guardian of each player. The testing was supervised by the first author at all venues. Wherever possible all tests were completed on the same day in the same order as described below:

1. body composition

2. $10 \mathrm{~m}$ and $40 \mathrm{~m}$ speed

3. Illinois agility test

4. 1RM bench press

5. underhand pull ups

6. push-ups

7. multistage shuttle run.

For various reasons beyond our control, some of the subjects did not complete all the tests. This is reflected in the sample size when the data are displayed.

\section{Body composition}

Body mass was recorded on a calibrated scale (Seca model 708, Hamburg, Germany) and recorded to the nearest $0.1 \mathrm{~kg}$. The players were weighed in underpants and without shoes. The stature of each player was recorded to the nearest millimetre, using a stadiometer (Seca model 708, Hamburg, Germany). The triceps, biceps, subscapular, supra-iliac, calf, thigh and abdominal skinfold thicknesses were measured according to the procedures described by Ross and Marfell-Jones. ${ }^{20}$ Body fat was estimated as the sum of 7 skinfolds $(\mathrm{mm})$ and as a percentage of body mass. ${ }^{8}$

\section{2. $10 \mathrm{~m}$ and $40 \mathrm{~m}$ speed}

The warm-up before this test consisted of a minimum of 10 minutes of submaximal running, followed by an appropriate stretching regimen and some acceleration sprints to familiarise the player with the pacing. An electronic sprint timer with photo-electric sensors was set at a height of $1.25 \mathrm{~m}$ and placed at $10 \mathrm{~m}$ and $40 \mathrm{~m}$ intervals from the start line. The player was instructed to crouch in the start position, $30 \mathrm{~cm}$ from the start line, after which he sprinted maximally for $40 \mathrm{~m}$ through the sensors. The player completed two maximal effort runs separated by a 5 - 10 minute recovery period. If the player was tested on a grass surface boots were worn, but no starting blocks were allowed. When testing occurred indoors, running shoes without spikes were used. The fastest $10 \mathrm{~m}$ and $40 \mathrm{~m}$ times for each player were recorded.

\section{Illinois agility test}

This test, modified from Getchell, ${ }^{14}$ measured the player's ability to accelerate, decelerate and change direction. The test started with the player lying in the prone position on the starting line with his chin touching the floor. On the signal of the whistle the player stood up and accelerated towards and around the cones, set up as prescribed for this test. ${ }^{14}$ The time taken to complete the course through the cones was recorded. The player had two attempts with a minimum rest period of 4 minutes between tests. The fastest time was recorded.

\section{1RM bench press}

The player lay supine on a bench with his feet flat on the floor and his hips and shoulders in contact with the bench. An Olympic bar was gripped $5-10 \mathrm{~cm}$ wider than shoulder width, using a closed pronated grip. The player started this test by lowering the bar in a controlled manner to the centre of the chest, touching the chest lightly and then extending upwards until the arms were in a fully locked position.

A light warm-up set of 10 repetitions was performed using a $20 \mathrm{~kg}$ weight. This was followed by $6-8$ repetitions at approximately $30-40 \%$ of the estimated $1 \mathrm{RM}$, which was based on the player's previous resistance training experience. A 2-minute stretching routine for the shoulders and chest was completed, followed by a further 6 repetitions on the bench press at a weight corresponding to $60 \%$ of the estimated 1RM. The player then rested for $3-4$ minutes before attempting his $1 \mathrm{RM}$. If the $1 \mathrm{RM}$ was successful, the player had a 5-minute rest before attempting a bench press using a resistance that had been increased by $2.5 \%$ to $5.0 \%$. Conversely, the resistance was decreased by $2.5 \%$ to $5.0 \%$ if the lift was not successful. The test was scored as the maximum weight $(\mathrm{kg})$ that could be lifted with one repetition. A lift was disqualified if the player raised his buttocks off the bench during the movement, bounced the bar off the chest, extended the arms unevenly, or if the spotter aided the lift. $1 \mathrm{RM}$ absolute bench press was recorded in kilograms $(\mathrm{kg})$, and the $1 \mathrm{RM}$ relative bench press was calculated as $1 \mathrm{RM} /$ (bodyweight $\left.{ }^{0.57}\right)^{7}$

\section{Underhand pull-ups}

The player started the test from a hanging position (arms fully extended) with the hands placed $10 \mathrm{~cm}$ apart in an underhand (supinated) grip. A valid pull-up required that the player's chin reached above the bar, and that at the end of each descent his arms were fully extended and his body remained stationary. The player continued the test until he could no longer lift himself to the bar. The test was scored as the number of valid pull-ups completed. 


\section{Push-ups}

The player began in a prone position with his hands on the floor, thumbs shoulder-width apart and elbows fully extended. Keeping the back and body straight the player descended to the tester's fist, placed on the floor below the player's sternum, and then ascended until the elbows were fully extended. If the player did not adhere to these specifications the repetition was not counted. The test was scored as the number of push-ups performed in 1 minute.

\section{Multistage shuttle run}

This progressive multistage shuttle run was based on the protocol of Lèger et al. ${ }^{15}$ A $20 \mathrm{~m}$ distance was measured out and marked on the floor. The players ran between these 2 lines. Players were instructed to complete each $20 \mathrm{~m}$ distance (lap) and turn according to the pace determined by the recorded sound signal. One foot of each player was required to touch the marked line, coinciding with the sound signal. The timing between signals started slowly and became progressively faster each minute. The players were warned if they failed to complete the $20 \mathrm{~m}$ distance in the required time for 2 consecutive laps. If this continued for the next lap they were withdrawn from the test. Players were also allowed to voluntarily withdraw from the test if they were unable to maintain the required pace. The score was recorded as the number of the last completed lap.

\section{Statistics}

Data are reported as means \pm standard deviations. A twoway analysis of variance was used to determine differences between age group and playing position for the different variables. When the overall F-value was significant for the main effect 'position', a Scheffe's post-hoc test was used to identify specific differences between positions. Statistical significance was accepted when $p<0.05$.

\section{Results}

There were no interactions between 'age' $X$ 'playing position' for any of the measurements.

The stature of 16-year-old players was significantly less than the 18-year-old players $(175.6 \pm 5.7$ v. $179.2 \pm 6.7 \mathrm{~cm}$; $p<0.001)$. Significant stature differences occurred between positions, with locks being taller than all other group positions (Table I).

Sixteen-year-old players weighed significantly less than the 18-year-old players $(76.5 \pm 8.2$ v. $84.9 \pm 8.3 \mathrm{~kg} ; p<$ $0.0001)$. Props were the heaviest and scrumhalves the lightest players in both age groups. Differences in body mass between positions are shown in Table II.

TABLE I. The stature $(\mathrm{cm})$ of 16 -year-old $(N=92)$ and 18-year-old $(N=82)$ rugby players*

\begin{tabular}{llrrr}
\hline Position & $\mathbf{1 6}$ years & $\boldsymbol{N}$ & $\mathbf{1 8}$ years & $\boldsymbol{N}$ \\
\hline Prop & $177.5 \pm 6.0$ & 10 & $180.3 \pm 3.8$ & 13 \\
Hooker & $173.4 \pm 3.1$ & 7 & $178.8 \pm 6.3$ & 5 \\
Lock & $187.2 \pm 5.5$ & 10 & $194.2 \pm 5.2$ & 11 \\
Loose forward & $180.8 \pm 4.3$ & 16 & $181.3 \pm 6.3$ & 15 \\
Scrumhalf & $165.9 \pm 10.3$ & 6 & $167.8 \pm 5.6$ & 8 \\
Flyhalf & $173.0 \pm 5.3$ & 8 & $177.6 \pm 7.6$ & 7 \\
Wing & $171.7 \pm 5.2$ & 15 & $176.4 \pm 8.2$ & 9 \\
Centre & $173.4 \pm 6.5$ & 12 & $179.1 \pm 8.5$ & 11 \\
Fullback & $178.1 \pm 5.5$ & 8 & $177.6 \pm 9.0$ & 3 \\
$\quad$ Average & $175.6 \pm 5.7$ & 92 & $179.2 \pm 6.7$ & 82 \\
& & & &
\end{tabular}

\section{Appropriate post-hoc analyses}

$\begin{array}{ll}\text { Position main effects } & \text { Significance } \\ \text { Props v. scrumhalf } & p<0.00002 \\ \text { Props v. wings } & p<0.05 \\ \text { Hookers v. scrumhalf } & p<0.015 \\ \text { Locks v. all positions } & p<0.00004 \\ \text { Loose forward v. scrumhalf } & p<0.00001 \\ \text { Loose forward v. wing } & p<0.0007 \\ \text { Scrumhalf v. flyhalf } & p<0.013 \\ \text { Scrumhalf v. centre } & p<0.003 \\ \text { Scrumhalf v. fullback } & p<0.0009\end{array}$

* The F-ratio was 12.56 for group $(p<0.001)$ and 19.8 for position $(p<0.0001)$ 
TABLE II. The body mass $(\mathrm{kg})$ of 16 -year-old $(N=92)$ and 18-year-old $(N=82)$ rugby players*

\begin{tabular}{llrlr}
\hline Position & $\mathbf{1 6}$ years & $\boldsymbol{N}$ & $\mathbf{1 8}$ years & $\boldsymbol{N}$ \\
\hline Prop & $95.5 \pm 14.1$ & 10 & $100.8 \pm 13.1$ & 13 \\
Hooker & $79.5 \pm 6.4$ & 7 & $93.1 \pm 5.7$ & 5 \\
Lock & $87.1 \pm 8.8$ & 10 & $95.2 \pm 8.4$ & 11 \\
Loose forward & $80.5 \pm 7.3$ & 16 & $88.2 \pm 5.5$ & 15 \\
Scrumhalf & $60.8 \pm 8.9$ & 6 & $70.3 \pm 4.9$ & 8 \\
Flyhalf & $69.6 \pm 5.3$ & 8 & $75.0 \pm 8.2$ & 7 \\
Wing & $68.4 \pm 6.7$ & 15 & $77.7 \pm 12.2$ & 9 \\
Centre & $71.9 \pm 9.1$ & 12 & $85.1 \pm 9.9$ & 11 \\
Fullback & $75.2 \pm 6.8$ & 8 & $78.8 \pm 6.6$ & 3 \\
Average & $76.5 \pm 8.2$ & 92 & $84.9 \pm 8.3$ & 82
\end{tabular}

\section{Appropriate post-hoc analyses}

$\begin{array}{ll}\text { Position main effects } & \text { Significance } \\ \text { Prop v. hooker } & p<0.00660 \\ \text { Prop v. loose forward } & p<0.00001 \\ \text { Prop v. scrumhalf } & p<0.00001 \\ \text { Prop v. flyhalf } & p<0.00001 \\ \text { Prop v. wing } & p<0.00001 \\ \text { Prop v. centre } & p<0.00001 \\ \text { Prop v. fullback } & p<0.00001 \\ \text { Hooker v. scrumhalf } & p<0.00001 \\ \text { Hooker v. flyhalf } & p<0.00889 \\ \text { Hooker v. wing } & p<0.00715 \\ \text { Lock v. scrumhalf } & p<0.00001 \\ \text { Lock v. flyhalf } & p<0.00001 \\ \text { Lock v. wing } & p<0.00001 \\ \text { Lock v. centre } & p<0.00006 \\ \text { Lock v. fullback } & p<0.00180 \\ \text { Loose forward v. scrumhalf } & p<0.00001 \\ \text { Loose forward v. flyhalf } & p<0.00521 \\ \text { Loose forward v. wing } & p<0.00005 \\ \text { Centre v. scrumhalf } & p<0.00975\end{array}$

* The F-ratio was 33.28 for group $(p<0.0001)$ and 24.84 for position $(p<0.0001)$
TABLE III. Sum of 7 skinfold measurements $(\mathrm{mm})$ for 16 -year-old $(N=91)$ and 18-year-old $(N=79)$ rugby players*

\begin{tabular}{llrlr}
\hline Position & 16 years & $\boldsymbol{N}$ & 18 years & $\boldsymbol{N}$ \\
\hline Prop & $110.8 \pm 37.6$ & 10 & $119.6 \pm 42.8$ & 12 \\
Hooker & $78.2 \pm 17.2$ & 7 & $80.7 \pm 31.9$ & 5 \\
Lock & $73.6 \pm 32.8$ & 10 & $68.1 \pm 18.9$ & 10 \\
Loose forward & $64.7 \pm 19.6$ & 16 & $62.3 \pm 12.3$ & 14 \\
Scrumhalf & $54.0 \pm 18.0$ & 6 & $58.3 \pm 17.1$ & 8 \\
Flyhalf & $59.3 \pm 10.3$ & 8 & $57.2 \pm 13.6$ & 7 \\
Wing & $54.7 \pm 12.1$ & 14 & $57.5 \pm 8.5$ & 9 \\
Centre & $51.2 \pm 10.1$ & 12 & $63.7 \pm 20.5$ & 11 \\
Fullback & $54.8 \pm 15.2$ & 8 & $55.5 \pm 20.1$ & 3 \\
Average & $66.8 \pm 19.2$ & 91 & $69.2 \pm 20.6$ & 79
\end{tabular}

Appropriate post-hoc analyses

Position main effects Significance

Prop v. all positions

$p<0.00204$

* The F-ratio was 0.43 for group $(p=0.51)$ and 15.47 for position $(p<0.0001)$

TABLE IV. Per cent body fat for 16 -year-old $(N=91)$ and 18-year-old $(N=82)$ rugby players*

\begin{tabular}{llrlr}
\hline Position & $\mathbf{1 6}$ years & $\boldsymbol{N}$ & $\mathbf{1 8}$ years & $\boldsymbol{N}$ \\
\hline Prop & $20.0 \pm 5.5$ & 10 & $20.0 \pm 4.2$ & 13 \\
Hooker & $16.4 \pm 2.4$ & 7 & $15.3 \pm 3.7$ & 5 \\
Lock & $14.8 \pm 4.6$ & 10 & $14.2 \pm 2.0$ & 11 \\
Loose forward & $14.2 \pm 3.1$ & 16 & $13.9 \pm 1.8$ & 15 \\
Scrumhalf & $13.2 \pm 4.2$ & 6 & $12.9 \pm 2.7$ & 8 \\
Flyhalf & $13.6 \pm 2.0$ & 8 & $13.3 \pm 2.4$ & 7 \\
Wing & $13.3 \pm 2.9$ & 14 & $13.1 \pm 1.6$ & 9 \\
Centre & $12.0 \pm 1.8$ & 12 & $13.8 \pm 2.9$ & 11 \\
Fullback & $13.0 \pm 4.6$ & 8 & $12.1 \pm 3.4$ & 3 \\
Average & $14.5 \pm 3.4$ & 91 & $14.3 \pm 2.7$ & 82
\end{tabular}

Appropriate post-hoc analyses

$\begin{array}{ll}\text { Position main effects } & \text { Significance } \\ \text { Prop v. lock } & p<0.00001 \\ \text { Prop v. loose forward } & p<0.00001 \\ \text { Prop v.slcrumhal } & p<0.00001 \\ \text { Prop v. flyhalf } & p<0.00001 \\ \text { Prop v. wing } & p<0.00001 \\ \text { Prop v. centre } & p<0.00001 \\ \text { Prop v. fullback } & p<0.00001\end{array}$

* The F-ratio was 0.15 for group $(p=0.70)$ and 10.69 for position $(p<0.0001)$. 
TABLE V. Bench press (absolute; kg) results for 16 -year-old $(N=71)$ and 18 year old $(N=80)$ rugby players*

\begin{tabular}{llrlr}
\hline Position & $\mathbf{1 6}$ years & $\boldsymbol{N}$ & $\mathbf{1 8}$ years & $\boldsymbol{N}$ \\
\hline Prop & $97.5 \pm 16.9$ & 8 & $102.7 \pm 26.3$ & 13 \\
Hooker & $83.0 \pm 10.4$ & 5 & $107.0 \pm 4.5$ & 5 \\
Lock & $80.6 \pm 12.1$ & 9 & $95.0 \pm 15.8$ & 11 \\
Loose forward & $82.7 \pm 18.4$ & 11 & $101.4 \pm 21.3$ & 14 \\
Scrumhalf & $63.0 \pm 6.7$ & 5 & $81.9 \pm 13.1$ & 8 \\
Flyhalf & $73.0 \pm 9.7$ & 5 & $82.1 \pm 20.8$ & 7 \\
Wing & $69.6 \pm 7.8$ & 13 & $94.4 \pm 23.2$ & 8 \\
Centre & $72.2 \pm 18.4$ & 9 & $98.2 \pm 12.1$ & 11 \\
Fullback & $72.5 \pm 6.1$ & 6 & $95.0 \pm 13.2$ & 3 \\
Average & $77.1 \pm 11.8$ & 71 & $95.3 \pm 16.7$ & 80
\end{tabular}

Appropriate post-hoc analyses

$\begin{array}{ll}\text { Position main effects } & \text { Significance } \\ \text { Prop v. scrumhalf } & p<0.00231 \\ \text { Prop v. flyhalf } & p<0.02941 \\ \text { Prop v. wing } & p<0.00094 \\ & \\ \text { * The F-ratio was } 37.59 \text { for the group }(p<0.00001) \text { and } 4.00 \text { for position } \\ (p<0.00001) \text {. }\end{array}$

TABLE VII. Number of pull-ups performed by 16-yearold $(N=77)$ and 18-year-old $(N=75)$ rugby players*

\begin{tabular}{lcrcr}
\hline Position & $\mathbf{1 6}$ years & \multicolumn{1}{c}{$\boldsymbol{N}$} & $\mathbf{1 8}$ years & $\boldsymbol{N}$ \\
\hline Prop & $7 \pm 7$ & 9 & $11 \pm 6$ & 13 \\
Hooker & $9 \pm 4$ & 6 & $13 \pm 3$ & 5 \\
Lock & $7 \pm 5$ & 9 & $8 \pm 6$ & 9 \\
Loose forward & $10 \pm 5$ & 12 & $13 \pm 6$ & 13 \\
Scrumhalf & $12 \pm 4$ & 5 & $16 \pm 11$ & 6 \\
Flyhalf & $12 \pm 5$ & 6 & $10 \pm 2$ & 7 \\
Wing & $11 \pm 3$ & 14 & $13 \pm 4$ & 8 \\
Centre & $13 \pm 4$ & 9 & $13 \pm 4$ & 11 \\
Fullback & $10 \pm 3$ & 7 & $13 \pm 3$ & 3 \\
Average & $10 \pm 5$ & 77 & $11 \pm 6$ & 75
\end{tabular}

\section{Appropriate post-hoc analyses}

$\begin{array}{ll}\text { Position main effects } & \text { Significance } \\ \text { Prop v. scrumhalf } & p<0.009 \\ \text { Prop v. wing } & p<0.02 \\ \text { Prop v. center } & p<0.006 \\ \text { Lock v. scrumhalf } & p<0.03\end{array}$

* The F-ratio was 2.8 for group $(p=0.10)$ and 3.8 for position $(p<0.001)$.
TABLE VI. Bench press (relative) results for 16-yearold $(N=71)$ and 18 -year-old $(N=80)$ rugby players*

\begin{tabular}{llccr}
\hline Position & 16 years & $\boldsymbol{N}$ & 18 years & $\boldsymbol{N}$ \\
\hline Prop & $7.39 \pm 1.08$ & 8 & $7.36 \pm 1.63$ & 13 \\
Hooker & $6.99 \pm 0.92$ & 5 & $8.09 \pm 0.51$ & 5 \\
Lock & $6.31 \pm 0.81$ & 9 & $7.09 \pm 1.12$ & 11 \\
Loose forward & $6.77 \pm 1.33$ & 11 & $7.86 \pm 1.53$ & 14 \\
Scrumhalf & $6.16 \pm 0.48$ & 5 & $7.25 \pm 1.12$ & 8 \\
Flyhalf & $6.58 \pm 0.70$ & 5 & $6.98 \pm 1.49$ & 7 \\
Wing & $6.28 \pm 0.70$ & 13 & $7.82 \pm 1.52$ & 8 \\
Centre & $6.35 \pm 1.36$ & 9 & $7.81 \pm 0.76$ & 11 \\
Fullback & $6.27 \pm 0.71$ & 6 & $7.81 \pm 0.76$ & 3 \\
Average & $6.55 \pm 1.00$ & 71 & $7.54 \pm 1.30$ & 80 \\
& & & & \\
* The F-ratio was 10.83 for group $(p<0.001)$ and 1.50 for position $(p<0.16)$. & \\
\end{tabular}

TABLE VIII. Number of push-ups performed by 16year-old $(N=84)$ and 18-year-old $(N=70)$ rugby players*.

\begin{tabular}{llrcr}
\hline Position & $\mathbf{1 6}$ years & $\boldsymbol{N}$ & $\mathbf{1 8}$ years & $\boldsymbol{N}$ \\
\hline Prop & $34 \pm 11$ & 10 & $46 \pm 18$ & 13 \\
Hooker & $44 \pm 14$ & 6 & $61 \pm 11$ & 5 \\
Lock & $41 \pm 14$ & 10 & $46 \pm 6$ & 8 \\
Loose forward & $42 \pm 14$ & 13 & $54 \pm 15$ & 13 \\
Scrumhalf & $44 \pm 9$ & 6 & $53 \pm 19$ & 6 \\
Flyhalf & $50 \pm 9$ & 6 & $56 \pm 17$ & 6 \\
Wing & $38 \pm 8$ & 15 & $50 \pm 16$ & 8 \\
Centre & $43 \pm 15$ & 11 & $56 \pm 10$ & 10 \\
Fullback & $35 \pm 7$ & 7 & $45 \pm 0.0$ & 1 \\
Average & $41 \pm 12$ & 84 & $52 \pm 15$ & 70 \\
& & & & \\
* The F-ratio was 16.6 for group $(p<0.000009)$ and 1.8 for position $(p=0.08)$. \\
\hline
\end{tabular}

Neither the sum of 7 skinfold measurements (Table III) nor body fat percentage (Table IV) were different between 16- and 18-year-old rugby players. There were significant differences between positions, however, with props having a greater percentage of body fat and higher skinfold measurement than the other positions (Tables III and IV).

There were significant differences between 16- and 18year-old groups regarding absolute and relative bench press measures (Tables $\mathrm{V}$ and $\mathrm{VI}$ ). The 16-year-old players lifted $77.1 \pm 11.8 \mathrm{~kg}(6.55 \pm 1.00$ relative to adjusted body mass) while the 18-year-old group lifted $95.3 \pm 16.7 \mathrm{~kg}(7.54 \pm$ 1.30 relative to adjusted body mass). There were significant position differences in absolute bench press measures between props versus scrumhalves, flyhalves and wings, with the props displaying greater upper body strength for 1RM. 
TABLE IX. 10 meter speed (seconds) of 16-year-old $(N=79)$ and 18-year-old $(N=72)$ rugby players*

\begin{tabular}{lrrrr}
\hline Position & $\mathbf{1 6}$ years & $\boldsymbol{N}$ & $\mathbf{1 8}$ years & $\mathbf{N}$ \\
\hline Prop & $2.0 \pm 0.1$ & 10 & $2.0 \pm 0.1$ & 11 \\
Hooker & $1.9 \pm 0.1$ & 7 & $1.9 \pm 0.1$ & 4 \\
Lock & $1.9 \pm 0.1$ & 8 & $1.9 \pm 0.1$ & 10 \\
Loose forward & $1.9 \pm 0.1$ & 15 & $1.9 \pm 0.1$ & 13 \\
Scrumhalf & $1.9 \pm 0.1$ & 4 & $1.8 \pm 0.0$ & 8 \\
Flyhalf & $1.9 \pm 0.1$ & 5 & $1.9 \pm 0.1$ & 6 \\
Wing & $1.8 \pm 0.1$ & 14 & $1.8 \pm 0.1$ & 9 \\
Centre & $1.8 \pm 0.1$ & 10 & $1.8 \pm 0.1$ & 8 \\
Fullback & $1.8 \pm 0.1$ & 6 & $1.8 \pm 0.1$ & 3 \\
Average & $1.9 \pm 0.1$ & 79 & $1.9 \pm 0.1$ & 72
\end{tabular}

\section{Appropriate post-hoc analyses}

$\begin{array}{ll}\text { Position main effects } & \text { Significance } \\ \text { Prop v. lock } & p<0.003 \\ \text { Prop v. loose forward } & p<0.00002 \\ \text { Prop v. scrumhalf } & p<0.00001 \\ \text { Prop v. flyhalf } & p<0.0002 \\ \text { Prop v. wing } & p<0.00001 \\ \text { Prop v. centre } & p<0.00001 \\ \text { Prop v. fullback } & p<0.00005 \\ \text { Lock v. wing } & p<0.05\end{array}$

* The F-ratio was 0.15 for group $(p=0.70)$ and 10.60 for position $(p<0.001)$.

Both age groups completed a similar number of pull-ups (10 \pm 5 v. $11 \pm 6$ repetitions for 16- v. 18-year-old groups). There were differences between props versus scrumhalves, wings and centres and between locks versus scrumhalves (Table VII). The 18-year-old players completed more pushups $(52 \pm 15)$ than the 16 -year-old players $(41 \pm 12 ; p<$ $0.000009)$. There were no differences between positions (Table VIII).

Speed was the same in both age groups when measured at 10 meters (1.9 \pm 0.1 seconds) and 40 meters (5.5 \pm 0.2 seconds). There were significant differences between positions, with props significantly slower than most other positions (Tables IX and $\mathrm{X}$ ). There were no differences in agility between the 16- and 18-year-old groups (Table XI). The props were less agile than scrumhalves, flyhalves, wings, centres and fullbacks.

In the multistage shuttle run the 18-year-old group ran significantly more shuttles than the 16-year-old group (93.5 \pm 15.3 v. $87.1 \pm 19.4$ shuttles; $p<0.05$ ). Props ran significantly fewer shuttles compared with loose forwards, scrumhalves, flyhalves and wings (Table XII).
TABLE X. 40 meter speed (seconds) of 16-year-old $(N=79)$ and 18-year-old $(N=73)$ rugby players*

\begin{tabular}{lrrrr}
\hline Position & $\mathbf{1 6}$ years & $\boldsymbol{N}$ & $\mathbf{1 8}$ years & $\boldsymbol{N}$ \\
\hline Prop & $5.8 \pm 0.1$ & 9 & $5.9 \pm 0.2$ & 11 \\
Hooker & $5.6 \pm 0.1$ & 7 & $5.5 \pm 0.4$ & 4 \\
Lock & $5.6 \pm 0.2$ & 8 & $5.6 \pm 0.2$ & 10 \\
Loose forward & $5.5 \pm 0.1$ & 15 & $5.5 \pm 0.2$ & 13 \\
Scrumhalf & $5.4 \pm 0.2$ & 5 & $5.4 \pm 0.2$ & 8 \\
Flyhalf & $5.4 \pm 0.1$ & 5 & $5.4 \pm 0.1$ & 6 \\
Wing & $5.3 \pm 0.2$ & 14 & $5.2 \pm 0.1$ & 9 \\
Centre & $5.3 \pm 0.0$ & 10 & $5.3 \pm 0.1$ & 9 \\
Fullback & $5.3 \pm 0.2$ & 6 & $5.3 \pm 0.1$ & 3 \\
Average & $5.5 \pm 0.2$ & 79 & $5.5 \pm 0.1$ & 73 \\
& & & &
\end{tabular}

$\begin{array}{ll}\text { Appropriate post-hoc analyses } & \\ \text { Position main effects } & \text { Significance } \\ \text { Prop v. all positions } & p<0.01 \\ \text { Hooker v. wing } & p<0.00005 \\ \text { Hooker v. centre } & p<0.002 \\ \text { Hooker v. fullback } & p<0.01 \\ \text { Lock v. scrumhalf } & p<0.01 \\ \text { Lock v. wing } & p<0.00001 \\ \text { Lock v. centre } & p<0.00001 \\ \text { Lock v. fullback } & p<0.003 \\ \text { Loose forward v. wing } & p<0.003 \\ \text { Loose forward v. centre } & p<0.05\end{array}$

* The F-ratio was 2.06 for group $(p=0.15)$ and 22.49 for position $(p<0.001)$

\section{Discussion}

The 16- and 18-year-old Green Squad players differed significantly in stature, body mass, arm strength (1 RM bench press and push-ups) and aerobic fitness (shuttle run) but not in percentage of body fat, speed $(10 \mathrm{~m}$ and $40 \mathrm{~m}$ ) or agility measures. These age group differences could be attributed to maturation, ${ }^{13}$ training discrepancies ${ }^{4}$ or a combination of the two.

The stature of the 16-year-olds $(175.6 \pm 5.7 \mathrm{~cm})$ and 18year-olds $(179.2 \pm 6.7 \mathrm{~cm})$ was shorter than those reported in junior rugby league players $(178 \mathrm{~cm}$ for 16 -year-olds and $182 \mathrm{~cm}$ for 17-year-olds) and college rugby league players $(181 \mathrm{~cm} \text { for } 20 \text {-year-old players })^{2}$ and amateur rugby union players $(184 \mathrm{~cm}){ }^{16}$

The body mass of the 16-year-old $(76.5 \pm 8.2 \mathrm{~kg})$ and 18 year-old $(84.9 \pm 8.3 \mathrm{~kg})$ players was heavier, ${ }^{11}$ lighter $^{2}$ and comparable ${ }^{4}$ with other junior rugby league players of similar ages. The 18-year-old Green Squad players were slightly heavier than amateur rugby players 20 years ago $(84.4 \mathrm{~kg}){ }^{16}$ 
TABLE XI. Illinois agility test results (seconds) for 16-year-old $(N=82)$ and 18-year-old $(N=50)$ rugby players*

\begin{tabular}{llrlr}
\hline Position & 16 years & $\boldsymbol{N}$ & 18 years & $\boldsymbol{N}$ \\
\hline Prop & $15.8 \pm 0.7$ & 10 & $16.3 \pm 1.2$ & 8 \\
Hooker & $15.2 \pm 0.8$ & 7 & $14.9 \pm 0.5$ & 3 \\
Lock & $15.5 \pm 0.9$ & 8 & $15.4 \pm 0.6$ & 9 \\
Loose forward & $15.6 \pm 0.9$ & 15 & $15.0 \pm 0.3$ & 6 \\
Scrumhalf & $14.6 \pm 0.5$ & 4 & $15.1 \pm 0.3$ & 6 \\
Flyhalf & $14.7 \pm 0.2$ & 6 & $14.5 \pm 0.4$ & 3 \\
Wing & $14.8 \pm 0.5$ & 15 & $14.4 \pm 0.2$ & 6 \\
Centre & $15.2 \pm 1.5$ & 11 & $14.4 \pm 0.4$ & 7 \\
Fullback & $14.7 \pm 0.5$ & 6 & $15.0 \pm 0.7$ & 2 \\
Average & $15.2 \pm 0.9$ & 82 & $15.1 \pm 0.8$ & 50
\end{tabular}

Appropriate post-hoc analyses

$\begin{array}{ll}\text { Position main effects } & \text { Significance } \\ \text { Prop v. scrumhalf } & p<0.04 \\ \text { Prop v. flyhalf } & p<0.007 \\ \text { Prop v.wing } & p<0.0002 \\ \text { Prop v. centre } & p<0.0007 \\ \text { Prop v. fullback } & p<0.04\end{array}$

* The F-ratio was 0.66 for group $(p=0.42)$ and 5.69 for position $(p<0.001)$

but obviously lighter than semi-professional and professional rugby players.

The 16-year-old group lifted less weight $(77.1 \pm 11.8$ $\mathrm{kg}$ ) than the 18-year-old group $(95.3 \pm 16.7)$ in the $1 \mathrm{RM}$ bench press test for upper body strength. This age-specific weakness was documented by Baker, ${ }^{2}$ where performance in the $1 \mathrm{RM}$ bench press was significantly correlated with playing achievement in untrained $(70.0 \mathrm{~kg})$, junior $(85.0 \mathrm{~kg})$, senior $(98.2 \mathrm{~kg})$, college $(110.5 \mathrm{~kg})$, and national $(144.5 \mathrm{~kg})$ level rugby league players (correlation coefficient $r=0.80$ ).

Upper body strength was further disparate, with 16-yearold players completing fewer push-ups $(41 \pm 12)$ than the 18year-old players $(52 \pm 15)$. These junior players performed more push-ups however, than US national team rugby players. (33). ${ }^{3}$

The 16-year-old group performed fewer shuttles (87.1 \pm 19.4; estimated $\mathrm{VO}_{2 \text { peak }} 49 \mathrm{ml} / \mathrm{kg} / \mathrm{min}$ ) than the 18-year-old group $\left(93.5 \pm 15.3\right.$; estimated $\left.\mathrm{VO}_{2 \text { peak }} 51 \mathrm{ml} / \mathrm{kg} / \mathrm{min}\right) .{ }^{15}$ This finding may be a result of maturation, where $\mathrm{VO}_{2}$ continues to increase through the age of $18,{ }^{13}$ combined with genetic limitations governing oxygen consumption. ${ }^{5}$ Training may also influence $\mathrm{VO}_{2 \max }$, contributing to approximately $35 \%$ of the variance of the increase. These measures of aerobic capacity are higher than those reported in junior and amateur rugby league players from Australia ${ }^{11,12}$ but lower than those
TABLE XII. The number of laps completed in the multistage shuttle run test for 16-year-old $(N=55)$ and 18-year-old $(N=63)$ rugby players*

\begin{tabular}{llrlr}
\hline Position & $\mathbf{1 6}$ years & $\boldsymbol{N}$ & $\mathbf{1 8}$ years & $\boldsymbol{N}$ \\
\hline Prop & $68.1 \pm 13.0$ & 9 & $77.6 \pm 11.1$ & 10 \\
Hooker & $89.0 \pm 7.6$ & 5 & $92.8 \pm 12.8$ & 4 \\
Lock & $89.8 \pm 22.6$ & 4 & $90.0 \pm 10.6$ & 9 \\
Loose forward & $97.5 \pm 24.0$ & 11 & $94.8 \pm 12.8$ & 9 \\
Scrumhalf & $85.7 \pm 5.7$ & 3 & $109.8 \pm 12.0$ & 6 \\
Flyhalf & $98.3 \pm 13.6$ & 3 & $98.7 \pm 14.3$ & 6 \\
Wing & $86.8 \pm 9.7$ & 8 & $99.9 \pm 23.6$ & 7 \\
Centre & $86.6 \pm 24.1$ & 9 & $93.0 \pm 10.1$ & 9 \\
Fullback & $92.0 \pm 18.4$ & 3 & $97.0 \pm 3.5$ & 3 \\
Average & $87.1 \pm 19.4$ & 55 & $93.5 \pm 15.3$ & 63 \\
& & & &
\end{tabular}

Appropriate post-hoc analyses

$\begin{array}{ll}\text { Position main effects } & \text { Significance } \\ \text { Prop v. loose forward } & p<0.0007 \\ \text { Prop v. scrumhalf } & p<0.03 \\ \text { Prop v. flyhalf } & p<0.03 \\ \text { Prop v. wing } & p<0.03\end{array}$

* The F-ratio was 4.22 for group $(p<0.05)$ and 3.83 for position $(p<0.001)$

values reported in other national team players. ${ }^{16}$

The number of pull-ups, sum of 7 skinfolds, per cent body fat, agility and speed were not significantly different between the two age groups. Of particular interest, both groups of players displayed the same average speed at $10 \mathrm{~m}(1.9$ seconds) and $40 \mathrm{~m}$ (5.5 seconds). These times are slower than those reported in professional rugby league players $(1.71 \text { and } 5.32 \mathrm{~s})^{1}$ but faster than those reported in Australian elite junior and semi-professional rugby league players. ${ }^{11,12}$

Positional differences were reported in measures of stature, body mass, sum of 7 skinfolds, per cent body fat, bench press (absolute), pull-ups, $10 \mathrm{~m}$ and $40 \mathrm{~m}$ speed, agility and aerobic fitness. A majority of these differences occurred between forward and back-line players with props having the most body fat, best upper body strength, slowest speed, least agility and lowest aerobic fitness of most other rugby players. These findings support another study which showed the props were the heaviest, slowest and least aerobically fit players, but outperformed all other players in measures of strength and power. ${ }^{19}$ This coincides with the particular demands of this position, where a high degree of body fat aids in absorbing impact during tackles and collisions while maximum strength and power are assets when competing for the ball in rucks, scrums and mauls. Heart rate and time motion analyses of under-19-year-old Australian rugby 
players indicate that front row (props and hookers) and back row (locks and loose forwards) forwards spend the most time in high exertion ( $58 \%$ and $56 \%$ respectively at $85-95 \% \mathrm{HR}_{\max }$ ) but cover the least distance (4 400 and $4080 \mathrm{~m}$ ) compared with inside (centres and flyhalves: $41 \%$ at $85-95 \% \mathrm{HR}_{\max }$, $5530 \mathrm{~m}$ ) and outside (wings and fullbacks: $34 \%$ at $85-95 \%$ $\mathrm{HR}_{\max }, 5750 \mathrm{~m}$ ) backs. ${ }^{6}$ These differences help explain the distinct physical characteristics necessary to succeed as a player in a particular position, thereby contributing to team success.

Conversely, backs are the shortest and lightest of rugby players. Inside, midfield and outside backs possess superior speed and agility to move the ball forward, accelerate away from defenders and out-manoeuvre opponents. ${ }^{9}$ These criteria are supported in our findings where backs are significantly faster, more agile and aerobically fit than forwards, particularly when compared with props.

It is also of interest to note that locks were significantly taller than all other rugby positions. This finding is consistent with other reports and exemplifies the specific demands placed on these players to jump to receive balls at lineouts. ${ }^{9}$

In conclusion, there are differences between elite 16- and 18-year-old rugby players with respect to stature, body mass, arm strength (1 RM bench press and push-ups) and aerobic fitness (shuttle run) but not in percentage of body fat, speed $(10 \mathrm{~m}$ and $40 \mathrm{~m})$ or agility measures. Positional differences exist, with props having the most body fat, best upper body strength, slowest speed, least agility and lowest aerobic fitness of most other rugby players.

This descriptive report provides a template for the evaluation of junior rugby players as well as baseline data for further study on the developmental characteristics and physiological progression of elite rugby athletes.

\section{Acknowledgements}

This study was funded by the South African Rugby Green Squad programme and Discovery Health, the Medical Research Council of South Africa and the Nellie Atkinson and Harry Crossley Research Funds of the University of Cape Town.

\section{REFERENCES}

1. Baker D, Nance $S$. The relation between running speed and measures of strength and power in professional rugby league players. J Strength Cond Res 1999; 13: 230 - 5.

2. Baker $D$. Differences in strength and power among junior-high, senior-high, college-aged, and elite professional rugby league players. J Strength Cond Res 2002; 16: 581-5.

3. Carlson BR, Carter JE, Patterson P, Petti K, Orfanos SM, Noffal GJ. Physique and motor performance characteristics of US national rugby players. J Sports Sci 1994; 12: 403-12.

4. Coutts AJ, Murphy AJ, Dascombe BJ. Effect of direct supervision of a strength coach on measures of muscular strength and power in young rugby league players. J Strength Cond Res 2004; 18: 316-23.

5. Danis $A$, Kyriazis $Y$, Klissouras $\mathrm{V}$. The effect of training in male prepubertal and pubertal monozygotic twins. Eur J Appl Physiol Occup Physiol 2003; 89: 309-18.

6. Deutsch MU, Maw GJ, Jenkins D, Reaburn P. Heart rate, blood lactate and kinematic data of elite colts (under-19) rugby union players during competition. J Sports Sci 1998; 16: 561-570.

7. Dooman CS, Vanderburgh PM. Allometric modeling of the bench press and squat: who is the strongest regardless of bodymass? J Strength Cond Res 2000; 14: 32-6.

8. Durnin JVGA, Womersley J. Body fat assessed from total body density and its estimation from skinfold thickness: measurements on 481 men and women aged from 16 to 72 years. Brit J Nutr 1974; 32: 77-97.

9. Duthie G, Pyne D, Hooper S. Applied physiology and game analysis of rugby union. Sports Med 2003; 33: 973-91.

10. Duthie GM. A framework for the physical development of elite rugby union players. International Journal of Sports Physiology and Performance 2006; 1: 2-13.

11. Gabbett TJ. Physiological characteristics of junior and senior rugby league players. Br J Sports Med 2002a; 36: 334-9.

12. Gabbett TJ. Influence of physiological characteristics on selection in a semi-professional first grade rugby league team: a case study. J Sports Sci 2000b; 20: 399-405.

13. Geithner CA, Thomis MA, Van den Eynde B, et al. Growth in peak aerobic power during adolescence. Med Sci Sports Exerc 2004; 36: 1616-24.

14. Getchell B. Physical Fitness: A Way of Life, 2nd ed. New York: John Wiley and Sons, Inc., 1979.

15. Lèger LA, Mercier D, Gadoury C, Lambert J. The multistage 20 metre shuttle run test for aerobic fitness. J Sports Sci 1988: 6: 93-101.

16. Maud PJ. Physiological and anthropometric parameters that describe a rugby union team. Br J Sports Med 1983: 17: 16-23.

17. Pienaar AE, Spamer MJ, Steyn HS jun. Identifying and developing rugby talent among 10-year-old boys: a practical model. J Sports Sci 1998; 16: 691-9.

18. Quarrie KL, Handcock P, Waller AE, Chalmers DJ, Toomey MJ, Wilson BD. The New Zealand rugby injury and performance project. III. Anthropometric and physical performance characteristics of players. Br J Sports Med 1995; 29: 263-70.

19. Quarrie KL, Handcock P, Toomey MJ, Waller AE. The New Zealand rugby injury and performance project. IV. Anthropometric and physical performance comparisons between positional categories of senior A rugby players. Br J Sports Med 1996; 30: 53-6.

20. Ross WD, Marfell-Jones MJ. Kinanthropometry. In: MacDougall JD, Wenger HA, Green HJ, eds. Physiological Testing of the High-Performance Athlete. Champaign, IL, USA: Human Kinetics Books, 1991, 223-308. 